\title{
Perceived Fatigue Impact and Cognitive Variability in Multiple Sclerosis
}

\author{
Kaitlin E. Riegler ${ }^{1} *$ (D) , Margaret Cadden ${ }^{1,2}$, Erin T. Guty ${ }^{1}$ (D) , Jared M. Bruce ${ }^{3}$ and Peter A. Arnett ${ }^{1}$ (D) \\ ${ }^{1}$ Department of Psychology, the Pennsylvania State University, State College, PA 16801, USA \\ ${ }^{2}$ Department of Neurology, Harvard Medical School, Brigham and Women's Hospital, Boston, MA 02115, USA \\ ${ }^{3}$ Department of Biomedical and Health Informatics, University of Missouri-Kansas City School of Medicine, Kansas City, MI 64108, USA
}

(Received October 14, 2020; Final Revision January 7, 2021; Accepted February 5, 2021; First Published Online March 31, 2021)

\begin{abstract}
Objective: People with Multiple Sclerosis (PwMS) and healthy controls (HCs) were evaluated on cognitive variability indices and we examined the relationship between fatigue and cognitive variability between these groups.

Intraindividual variability (IIV) on a neuropsychological test battery was hypothesized to mediate the group differences expected in fatigue. Method: Fifty-nine PwMS and $51 \mathrm{HCs}$ completed a psychosocial interview and battery of neuropsychological tests and questionnaires during a 1-day visit. Fatigue in this study was measured with the Fatigue Impact Scale (FIS), a self-report multidimensional measure of fatigue. IIV was operationalized using two different measures, a maximum discrepancy score (MDS) and intraindividual standard deviation (ISD), in two cognitive domains, memory and attention/processing speed. Two mediation analyses with group (PwMS or HCs) as the independent variable, variability composite (memory or attention/processing speed) measures as the mediators, total residual fatigue (after accounting for age) as the outcome, and depression as a covariate were conducted. The Baron and Kenny approach to testing mediation and the PROCESS macro for testing the strength of the indirect effect were used.

Results: Results of a mediation analysis using 5000 bootstrap samples indicated that IIV in domains of both attention/ processing speed and memory significantly mediated the effect of patient status on total residual fatigue. Conclusion: IIV is an objective performance measure that is related to differences in fatigue impact between PwMS and HCs. PwMS experience more variability across tests of attention/processing speed and memory and this experience of variable performance may increase the impact of fatigue.
\end{abstract}

Keywords: Cognition, Self-report, Neuropsychological tests, Neurodegenerative diseases, Cognitive dysfunction, Central nervous system

\section{INTRODUCTION}

Fatigue is a debilitating and prevalent experience for people with Multiple Sclerosis (PwMS) that can exacerbate other symptoms of MS (Fisk, Pontefract, Ritvo, Archibalnd, \& Murray, 1994; Freal, Kraft, \& Coryell, 1984; Homorodean, Leucuta, \& Perju-Dumbravă, 2016; MacAllister \& Krupp, 2005). Between 76 and $97 \%$ of PwMS report fatigue as one of their symptoms (van Kessel \& Moss-Morris, 2006) and $40 \%$ of PwMS report fatigue as their most disabling symptom (MacAllister \& Krupp, 2005). Fatigue in MS worsens over the course of the day and can be aggravated or worsened by heat and stress among other factors (Freal

*Correspondence and reprint requests to: Kaitlin Riegler, Department of Psychology, 372 Moore Building, the Pennsylvania State University, University Park, PA 16802, USA. E-mail: kriegler25@gmail.com et al., 1984; Schwartz, Coulthard-Morris, \& Zeng, 1996). MS-related fatigue is distinctly different from other types of fatigue. It tends to be more persistent, comes on easily, and can prevent sustained physical functioning (Homorodean et al., 2016). As such, the Multiple Sclerosis Council for Clinical Practice Guidelines has defined fatigue in the context of MS as a "subjective lack of physical and/or mental energy that is perceived by the individual or caregiver to interfere with usual and desired activities" (Multiple Sclerosis Council For Clinical Practice Guidelines, 1998). It has been suggested that secondary factors common in MS, such as sleep disturbance and mood difficulties, are involved in MS-related fatigue (Krupp, Serafin, \& Christodoulou, 2010; Strober \& Arnett, 2005). The disruption in daily life caused by this fatigue can manifest as interference with physical activities and difficulty with activities of daily living. 
It also contributes to problems maintaining full-time employment (Strober et al., 2018) and is sometimes associated with lapses in memory, concentration, and other important cognitive processes (Cadden \& Arnett, 2015; Jongbloed, 1998; Strober, Chiaravalloti, \& DeLuca, 2018).

\section{FATIGUE AND FATIGUABILITY}

Across the literature, many terms have been used to refer to different aspects or components of fatigue, including central fatigue, peripheral fatigue, domains of fatigue (e.g., cognitive and physical fatigue), and performance fatiguability. Oftentimes, some of these terms are not clearly defined or operationalized. This occurs despite evidence that perceived fatigue and fatiguability are distinct constructs (Kluger, Krupp, \& Enoka, 2013). Perceived fatigue is the subjective experience of fatigue and is often collected using self-report measures of fatigue. This may include the subjective experience of weariness, an increase in effort, or an experienced mismatch between the effort being exerted and the actual outcome or performance (DeLuca, 2005; Kluger et al., 2013). Fatiguability, on the other hand, is typically measured as the rate of change in performance over a period of time, usually relative to a control or some other reference value, and is thought to be a more objective measurement of fatigue (Charvet, Serafin, \& Krupp, 2014; Kluger et al., 2013). Fatiguability tends to be most associated with the domain of cognitive fatigue. Fatiguability has been examined utilizing several paradigms including where cognitive fatigue is created via effortful mental tasks, and then performance on neuropsychological measures is compared before and after this effortful task, by comparing performance at the end of a single task to performance at the beginning of that same task, or by observing performance decrement on motor tasks over time.

\section{Perceived fatigue and cognitive dysfunction}

While there is some evidence that fatigue, in healthy individuals, is correlated with neuropsychological test performance in a number of different domains including concentration, verbal memory, speed of information processing, and motor speed (Busichio, Tiersky, Deluca, \& Natelson, 2004; Cockshell \& Mathias, 2010; Johnson, Lange, DeLuca, Korn, \& Natelson, 1997; Lezak, Howieson, Bigler, \& Tranel, 2012), the literature in PwMS specifically has been mixed regarding the impact of self-reported fatigue on neuropsychological test performance. Most studies report no association between subjective reports of fatigue and cognitive deficits (Bryant, Chiaravalloti, \& DeLuca, 2004; DeLuca, 2005; Krupp et al., 2010; Parmenter, Denney, \& Lynch, 2003; Paul, Beatty, Schneider, Blanco, \& Hames, 1998), but some studies report fatigue-related differences in cognitive performance (Bruce, Bruce, \& Arnett, 2010; Hanken, Eling, \& Hildebrandt, 2015; Krupp et al., 2010; Pokryszko-Dragan et al., 2016).

\section{Fatiguability and cognitive dysfunction}

Research on the association between fatiguability and cognitive performance has been somewhat more conclusive, with the majority of studies demonstrating that fatiguability (e.g., cognitive exertion) impairs cognitive performance over time (Johnson et al., 1997; Krupp \& Elkins, 2000; Kujala, Portin, Revonsuo, \& Ruutiainen, 1995). One group of researchers has examined the neural correlates of diminished or variable performance during sustained effort over time using performance on a modified Symbol Digit Modalities (mSDMT) test as a marker of state mental fatigue (Chen et al., 2020; DeLuca, Genova, Hillary, \& Wylie, 2008). Results from this series of studies demonstrated that state mental fatigue was associated with greater activation in the caudate in PwMS compared to controls and that PwMS allocated neural resources less efficiently when sustaining effort over time. Another study comparing PwMS to control participants on neuropsychological test performance revealed that PwMS had a larger decline in cognitive performance than controls following cognitive effort on indices of visual memory, verbal memory, and verbal fluency (Krupp \& Elkins, 2000).

\section{VARIABILITY IN PERFORMANCE}

Researchers have hypothesized that the lack of consensus in the literature around the role of fatigue on cognitive performance in MS may be the result of differences in categorization or definition of fatigue, differences in methods employed to "invoke" fatiguing conditions, and method of performance measurement. We argue that the mixed findings tying reported fatigue to objective performance deficits may be the result of the method of performance measurement. Most of the literature has focused on mean differences before and after a period of mental exertion (effortful cognitive tasks). One previous study by Bryant and colleagues (2004) suggested that different performance measures may be reflective of different underlying mechanisms, thus highlighting the importance of using more than just mean-level differences. Another study found that measures of performance strategy, rather than pure performance measures, are more sensitive to cognitive fatigue in PwMS than HCs (Bryant et al., 2004). Others have examined changes in intraindividual variability (IIV) over time and determined that this might be a more ecologically valid metric for measuring fatiguability and the impact on cognitive performance (Wang, Ding, \& Kluger, 2014). Taken together, this suggests that measuring cognitive impairment at the intraindividual level, rather than examining group-level mean differences, might be particularly illuminating when considering the impact of fatigue in MS. Further, IIV may be conceptualized as a measurement of fatiguability over a testing session.

In a variety of clinical samples, it has been established that IIV may be a better predictor of cognitive outcome than mean differences (Burton, Strauss, Hultsch, Moll, \& Hunter, 2006; Cole, Weinberger, \& Dickinson, 2011; Haynes, Bauermeister, \& Bunce, 2017; MacDonald, Nyberg, \& 
Bäckman, 2006). While more limited, there is some literature on the use of variability as a performance-based measure thought to reflect the impact of fatigue on performance over a period of time in MS (Bruce et al., 2010; Krupp et al., 2010). Bruce and colleagues (2010) examined the association between self-reported cognitive fatigue and a measure of response time variability (RTV). PwMS demonstrated higher RTV compared to HCs and RTV was highly correlated with cognitive fatigue (Bruce et al., 2010). Another study examined RTV in MS as a performance-based measure of fatiguability and demonstrated that performance on an attention task was significantly associated with perceived physical and cognitive fatigue (Aldughmi, Bruce, \& Siengsukon, 2017). Holtzer et al. (2013) employed burst measurement of the SDMT and found increased variability in processing speed across repeated administrations of the SDMT. Wojowicz and colleagues (2012) compared PwMS to HCs and PwMS demonstrated greater IIV, measured by an individual standard deviation score, on all subtests of the Computerized Test of Information Processing (Wojtowicz, Berrigan, \& Fisk, 2012). Group differences in cognitive impairments may not fully capture the subtle differences that can be the result of MS-related fatigue; as such, indices of variability may be more reflective of the impact of fatigue.

\section{CURRENT STUDY}

With these considerations in mind, the goal of the current study was to evaluate differences between PwMS and HCs on cognitive variability indices and examine the relationship between self-report measures of fatigue and cognitive variability between these groups. IIV was operationalized using two different measures, a maximum discrepancy score (MDS) and intraindividual standard deviation (ISD), in two cognitive domains, memory and attention/processing speed. IIV was hypothesized to mediate the group differences expected in total reported fatigue. These data are crosssectional, and therefore it is difficult to establish the directionality of the mediation. Therefore, we will also test the reverse mediation, whereby total reported fatigue is hypothesized to mediate the group differences in IIV.

\section{METHODS}

\section{Procedure}

This study involved an analysis of data collected as part of a project examining cognitive, motor, and emotional factors in MS. Analyses from this project were run on data collected as part of this study (Arnett, Smith, Barwick, Benedict, \& Ahlstrom, 2008). Participants completed a psychosocial interview and a battery of neuropsychological tests and questionnaires during a 1-day visit. This study was approved by the Institutional Review Board at the Pennsylvania State University and all participants signed informed consent prior to participating in the study.

\section{Participants}

\section{MS group}

This study included 59 PwMS ( $M=10, F=49)$. The mean age was 51.63 and the mean disease duration was 14.90 years. Participants for this study were recruited from the greater Central Pennsylvania area. Thompson et al.'s (2017) revised McDonald criteria were applied to determine a positive diagnosis of MS (Thompson et al., 2018). Exclusion criteria were as follows: significant history of substance abuse, nervous system disorder other than MS, sensory impairment that could interfere with testing, developmental history of attention-deficit hyperactivity disorder (ADHD) or learning disability, significant medical condition other than MS that could interfere with cognitive or motor function, relapse or corticosteroid use within 4 weeks of participation in the study, or physical or neurological impairment that would make the testing impossible. MS course types included relapsing-remitting $(n=35)$, secondary progressive $(n=17)$, and primary progressive $(n=7)$. All participants were paid $\$ 100$ for their participation.

\section{Healthy control group}

This study included 51 neurologically healthy communitybased individuals $(\mathrm{M}=8, \mathrm{~F}=43)$. HCs were matched as closely as possible to the MS participants on demographic factors such as age, education, and gender. HCs were recruited using several methods: MS participants were asked to recommend friends, advertisements were posted in public places in Central Pennsylvania, and an ad was posted on the university newswire. HCs were also paid $\$ 100$ for their participation. See Table 1 for participant demographic characteristics.

\section{Measures}

\section{Fatigue}

The Fatigue Impact Scale (FIS) is a 40-item self-report measure of fatigue that measures different types of fatigue including cognitive (e.g., "I have been less alert"), physical (e.g. "My muscles have felt weak"), and psychosocial components (e.g., "I have been limited in my ability to do things away from home") (Fisk, Ritvo, et al., 1994). Items are rated on a 5-point Likert scale of how much of a problem fatigue has caused in the past month 0 (no problem) to 4 (extreme problem). This self-report measure has been shown to have good psychometric properties and to be sensitive to changes in fatigue in PwMS (Whitehead, 2009). The three FIS scales were highly correlated in our sample, $r=.64-.85$, and thus the total fatigue score from this scale was used as our outcome variable.

\section{Depression}

Depression was measured using the Beck Depression Inventory-Fast Screen (BDI-FS) (Beck, Steer, \& Brown, 2000). The BDI-FS is a commonly used brief self-report 
Table 1. Demographic characteristics of PwMS and HC groups

\begin{tabular}{|c|c|c|c|c|c|c|c|}
\hline & \multicolumn{3}{|c|}{ PwMS } & \multicolumn{3}{|c|}{$\mathrm{HC}$} & \multirow[b]{2}{*}{$p$} \\
\hline & Mean & $S D$ & Range & Mean & $S D$ & Range & \\
\hline$\%$ Female & 83.1 & - & - & 84.3 & - & - & - \\
\hline$\%$ Caucasian & 100 & - & - & 96.1 & - & - & - \\
\hline Age (years) & 51.63 & 9.45 & $31-70$ & 46.22 & 10.77 & $25-67$ & .006 \\
\hline EDSS & 4.08 & 2.08 & $0-7.5$ & - & - & - & - \\
\hline Disease duration (years) & 14.90 & 8.57 & $3.75-40.25$ & - & - & - & - \\
\hline FIS total score & 56.88 & 30.15 & $0-118$ & 13.38 & 13.45 & $0-59$ & $<.001$ \\
\hline FIS cognitive scale & 13.11 & 8.05 & $0-33$ & 4.98 & 5.32 & $0-25$ & $<.001$ \\
\hline FIS physical scale & 18.40 & 9.73 & $0-40$ & 3.10 & 3.57 & $0-15$ & $<.001$ \\
\hline FIS social scale & 25.40 & 15.63 & $0-59$ & 5.94 & 7.04 & $0-36$ & $<.001$ \\
\hline WTAR FSIQ & 105.72 & 8.00 & $80-118$ & 107.73 & 7.35 & $90-118$ & .18 \\
\hline BDI-FS & 2.64 & 2.40 & $0-13$ & .78 & 1.21 & $0-5$ & $<.001$ \\
\hline $\begin{array}{l}\text { Attention/Processing speed } \\
\text { variability composite }\end{array}$ & 95.61 & 13.79 & $56.41-131.85$ & 104.38 & 11.27 & $75.76-139.07$ & $<.001$ \\
\hline Memory variability composite & 96.60 & 13.62 & $53.77-124.27$ & 103.95 & 15.35 & $68.55-129.15$ & .009 \\
\hline
\end{tabular}

BDI-FS = Beck Depression Inventory-Fast Screen; EDSS = Expanded Disability Status Scale; FIS = Fatigue Impact Scale; HCs = Healthy Controls; PwMS = Persons with Multiple Sclerosis; WTAR FSIQ = Wechsler Test of Adult Reading Full-Scale IQ.

measure of depression in medical populations. Previous work has identified the BDI-FS as a good screen for depression in MS since it excludes neurovegetative symptoms that commonly overlap with symptoms of MS (Benedict, Fishman, McClellan, Bakshi, \& Weinstock-Guttman, 2003; Strober \& Arnett, 2015). It includes seven items that examinees rate based on how they have felt over the past 2 weeks. Each item has four statements that are assigned a value of 0 through 3 , with lower scores indicating lower depression symptomatology.

\section{Neuropsychological test battery}

The neuropsychological test battery included the following measures: Wechsler Test of Adult Reading (WTAR; The Psychological Corporation, 2001), Digits Forward, Digits Backward (Weschler, 1997), Written Symbol Digit Modalities Test (SDMT), Oral SDMT (Shum, McFarland, \& Bain, 1990), Controlled Oral Word Association Test (COWAT) Total, Animal Naming Total (Delis, Kaplan, \& Kramer, 2001), Paced Auditory Serial Addition Task (PASAT) - 3-Second Trial Total Correct, PASAT 2-Second Trial Total Correct, Visual Elevator subtest from the Test of Everyday Attention Time Per Correct Switch (Robertson, Ward, Ridgeway, \& Nimmo-smith, 2001), 10/36 Spatial Recall Immediate Recall Total, 10/36 Spatial Recall Delayed Recall (Rao \& Cognitive Function Study Group, 1990), Brief Visuospatial Memory Test - Revised (BVMT-R) Total Immediate Recall, BVMT-R Delayed Recall (Benedict, 1997), and the California Verbal Learning Test-II (CVLT-II) Immediate Recall, CVLT-II Short Delay Recall, and CVLT-II Long-Delay Recall (Delis, Kramer, Kaplan, \& Ober, 2000).

Scores from each test index were transformed to standard scores with a mean of 100 and a standard deviation of
15 using the sample mean and standard deviation of the HCs. Scores were created such that higher scores always indicated better performance. Comparable to previous work in other populations (Guty \& Arnett, 2018; Riegler, Guty, $\&$ Arnett, 2019), after the scores were standardized, two composites were created based on these neuropsychological test indices by taking the mean of the standard scores of tests comprising that composite. A memory composite was comprised of the 10/36 Spatial Recall Immediate Total, the 10/36 Spatial Recall Delay Total, BVMT-R Total Immediate Recall, BVMT-R Delayed Recall, CVLT-II Immediate Recall, CVLT-II Short Delay Recall, and CVLT-II Long-Delay Recall. An attention/processing speed composite was comprised of Digits Forward, Digits Backward, SDMT Written, SDMT Oral, COWAT Total, Animal Naming Total, PASAT - 3-Second Trial Total Correct, PASAT 2-Second Trial Total Correct, and Visual Elevator Time Per Correct Switch. A principal components analysis was conducted for the tests in each composite and all of the variables loaded above .50 .

\section{Intraindividual cognitive variability indices}

Once scores were standardized, the cognitive variability indices were created. Two IIV indices were derived for each of the composites created above (memory and attention/ processing speed). Previous work has used the variability metric of MDS (Heyanka, Holster, \& Golden, 2013; Rabinowitz \& Arnett, 2013). This metric subtracts the lowest score from the highest score for each individual participant. Other studies of IIV have used ISD as a measure of variability across a test battery. This method takes the standard deviation of the Standard Scores across the test battery (Burton et al., 2006; Fuentes et al., 2001; Merritt et al., 2019). Based on this 
Table 2. Correlations among key study variables

\begin{tabular}{|c|c|c|c|c|c|c|}
\hline & Age & FIS total & BDI-FS & WTAR FSIQ & $\begin{array}{l}\text { Memory variability } \\
\text { composite }\end{array}$ & $\begin{array}{l}\text { Attention/processing speed } \\
\text { variability composite }\end{array}$ \\
\hline Age & 1 & $.32 * *$ & .17 & .03 & $.33 * *$ & $.43 * *$ \\
\hline FIS total & & 1 & $.72 * *$ & -.03 & $.24 *$ & $.36 * *$ \\
\hline BDI-FS & & & 1 & -.09 & .10 & $.31 * *$ \\
\hline WTAR FSIQ & & & & 1 & -.18 & $-.24 *$ \\
\hline Memory variability composite & & & & & 1 & $.25 * *$ \\
\hline $\begin{array}{l}\text { Attention/processing speed } \\
\text { variability composite }\end{array}$ & & & & & & 1 \\
\hline
\end{tabular}

BDI-FS = Beck Depression Inventory-Fast Screen; FIS = Fatigue Impact Scale; HCs = Healthy Controls; PwMS = Persons with Multiple Sclerosis; WTAR FSIQ $=$ Wechsler Test of Adult Reading Full-Scale IQ Estimate.

$*=p<.01, * *=p<.001$.

previous work, we calculated these two measures of IIV for participants in our study.

Initially, four indices were created: memory MDS, memory ISD, attention/processing speed MDS, and attention/processing speed ISD. There was a high degree of correlation between the variability indices for each composite so the two variability indices for each set of tests were combined to form one measure of IIV for each set of tests. Memory ISD was significantly correlated with memory MDS, $r(108)=0.96, p<.001$, and attention/processing speed ISD was significantly correlated with attention/ processing speed MDS, $r(108)=.77, p<.001$. The two IIV indices for each domain of tests were then standardized and a memory variability composite and attention/processing speed variability composite were created as the final two outcome measures to be used in mediation analyses.

\section{RESULTS}

\section{Data analysis}

All analyses were conducted with the Statistical Package for the Social Sciences (SPSS), Version 25.0 (IBM Corporation, 2017).

\section{Preliminary analyses}

Independent samples $t$ tests were conducted to compare demographic differences between PwMS and HCs. As shown in Table 1, PwMS were significantly older and reported significantly more depression and total fatigue. The groups did not differ on premorbid IQ as measured with the WTAR Full-Scale IQ (FSIQ) estimate. Next, bivariate correlation analyses among key variables of interest in this study were conducted (See Table 2). As seen in this table, age was significantly correlated with total fatigue scores and both IIV variables. Due to the age difference between the groups and the significant correlations between age and the mediators and outcome variables, age was included in the mediation model by regressing age on total fatigue and then using the unstandardized residuals (total residual fatigue) as the new outcome variable in the two mediation models.
Additionally, as seen in Table 2, depression was significantly correlated with one of the mediators (attention/processing speed IIV), $r=.31, p<.001$, and the outcome (total fatigue), $r=.72$, $p<.001$. Since depression is more common in PwMS than in the general population, it is an important defining characteristic of our MS group compared to the HCs; estimates range from 27 to 54\% of PwMS meeting criteria for clinically significant depression compared to $7-10 \%$ in the general population (American Psychiatric Association, 2013; Arnett, Barwick, \& Beeney, 2008). In our sample, 18 PwMS and $1 \mathrm{HC}$ met the cutoff for clinically significant depression $(\geq 4)$. Previous studies have reported small to moderate effects for the relationship between depression and fatigue (Arnett et al., 2008). Further, some of the symptoms of depression are similar to symptoms of fatigue in MS (Strober \& Arnett, 2010). To parse apart the contributions of fatigue separate from depression, depression was included as a covariate in mediation models.

\section{Hypothesis testing analyses: mediation}

Mediation analyses with group (MS or HC) as the independent variable, total age-corrected residual fatigue as the dependent variable, variability composite (memory and attention/processing speed IIV) measures as the mediators, and total BDI-FS scores as a covariate were conducted. The Preacher and Hayes approach was used to test mediation (Preacher \& Hayes, 2004). This method utilizes the PROCESS macro of SPSS and was used to test the strength of the indirect effect (Preacher \& Hayes, 2004). The indirect effect is the extent to which the dependent variable (total residual fatigue) changes when the independent variable is held fixed and the mediator (IIV) changes by the amount it would have changed had the independent variable (total residual fatigue) increased by one unit. The parameters used for the analyses of the PROCESS macro were Model 4, 5000 bootstrap samples, and a $95 \%$ bias-corrected confidence interval (CI).

Attention/processing speed variability composite as mediator. Regression analysis was used to investigate the hypothesis that attention/processing speed IIV mediates the 


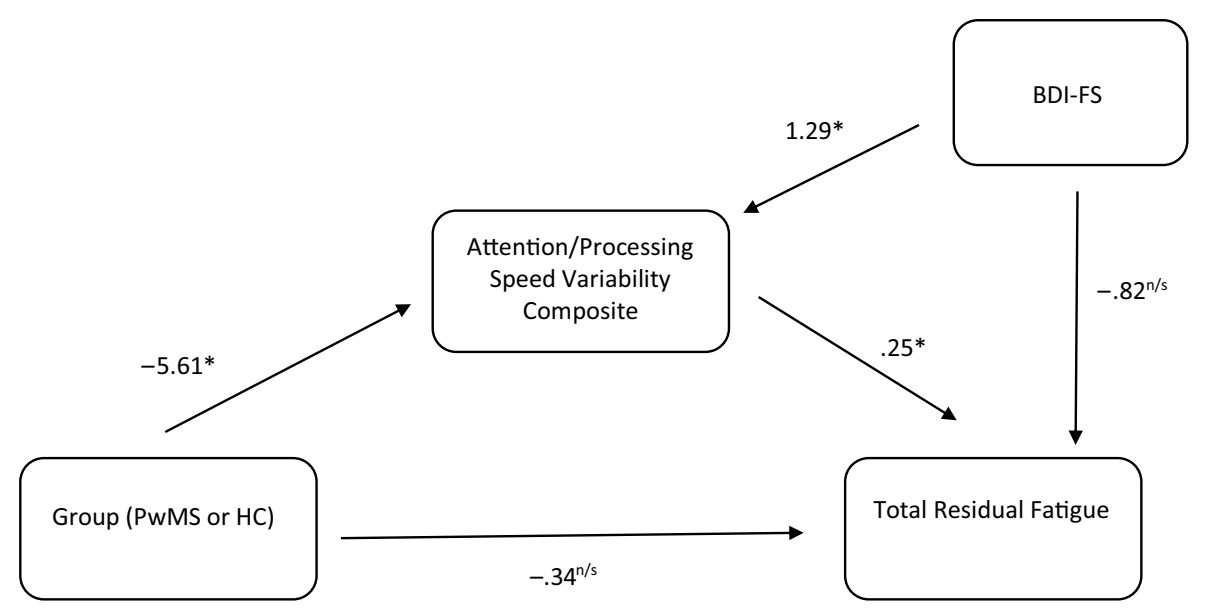

Fig. 1. Mediation model with attention/processing speed composite as mediator and depression as covariate. BDI-FS = Beck Depression Inventory-Fast Screen; HCs = Healthy Controls; PwMS = Persons with Multiple Sclerosis. $*=p<.05,{ }^{\mathrm{n} / \mathrm{s}}=p>.05$ (not significant).

effect of patient status on total residual fatigue after controlling for depression. Results indicate that, after controlling for depression, patient status was a significant predictor of attention/processing speed IIV, $b=-5.61, t(104)=$ $-2.14, p=.04$, and that attention/processing speed IIV was a significant predictor of total residual fatigue, $b=.25, t(103)=3.38, p=.001$. These results support the mediational hypothesis. Patient status was no longer a significant predictor of total residual fatigue after controlling for the mediator, attention/processing speed IIV, $b=-.34$, $t(103)=-.17, p=.87$. Results of a mediation analysis using 5000 bootstrap samples indicated that attention/processing speed IIV significantly mediated the increase in total residual fatigue in the MS group relative to the $\mathrm{HC}$ group, indirect effect $-1.42,95 \%[-3.09,-.09]$. PwMS demonstrate more attention/processing speed variability which leads to more self-reported fatigue. See Figure 1 for a graphical representation of the mediation model with attention/processing speed IIV as the mediator. When this relationship was tested in reverse, total residual fatigue did not significantly mediate the increase in attention/processing speed IIV in the MS group relative to the control group, indirect effect $=-.70$, 95\% CI [-2.51, 1.08].

Memory variability composite variability as mediator. Regression analysis was used to investigate the hypothesis that memory IIV mediates the effect of patient status on total residual fatigue after controlling for depression. Results indicate that, after controlling for depression, patient status was a significant predictor of memory IIV, $b=-7.37$, $t(104)=-2.33, p=.02$, and that memory IIV was a significant predictor of total residual fatigue, $b=.17, t(103)=2.76$, $p=.007$. These results support the mediational hypothesis. Patient status was no longer a significant predictor of total residual fatigue after controlling for the mediator, memory IIV $, b=-.48, t(103)=-.23, p=.82$. Results of a mediation analysis using 5000 bootstrap samples indicated that memory
IIV significantly mediated the increase in total residual fatigue in the MS group relative to the HC group, indirect effect - 1.29, 95\% [-2.71, -.08]. PwMS demonstrate more memory variability which leads to more self-reported fatigue. See Figure 2 for a graphical representation of the mediation model with memory IIV as the mediator. When this relationship was tested in reverse total residual fatigue did not significantly mediate the increase in memory IIV in the MS group relative to the control group, indirect effect $=-.70$, $95 \%$ CI $[-2.52,1.14]$.

\section{DISCUSSION}

The present study was designed to evaluate differences between PwMS and HCs on IIV indices and examine the relationship between fatigue and cognitive variability between these groups. IIV was hypothesized to mediate the group differences expected in total fatigue. Prior research on fatigue in PwMS has been mixed, with some studies reporting an association between perceived fatigue and cognitive performance and other studies reporting no association. Potential explanations for these mixed findings include both the method of cognitive performance measurement and the conceptualization of the mechanism of fatiguability. Previous studies have measured cognitive performance as mean-level differences before and after an effortful cognitive task, for example, repeated performance on the PASAT or an n-back task. This paradigm, therefore, assumes that cognitive exertion in and of itself evokes fatiguability and that fatiguability may be the mechanism by which fatigue impairs performance. That is, if performance after a period of cognitive exertion is worse than before in PwMS, this paradigm assumes that the cognitive exertion was fatigue causing and that this explains that decline in performance. We sought to further explore the relationship between perceived fatigue and performance in PwMS by utilizing an alternative performance-based measure, IIV, and a mediation model 


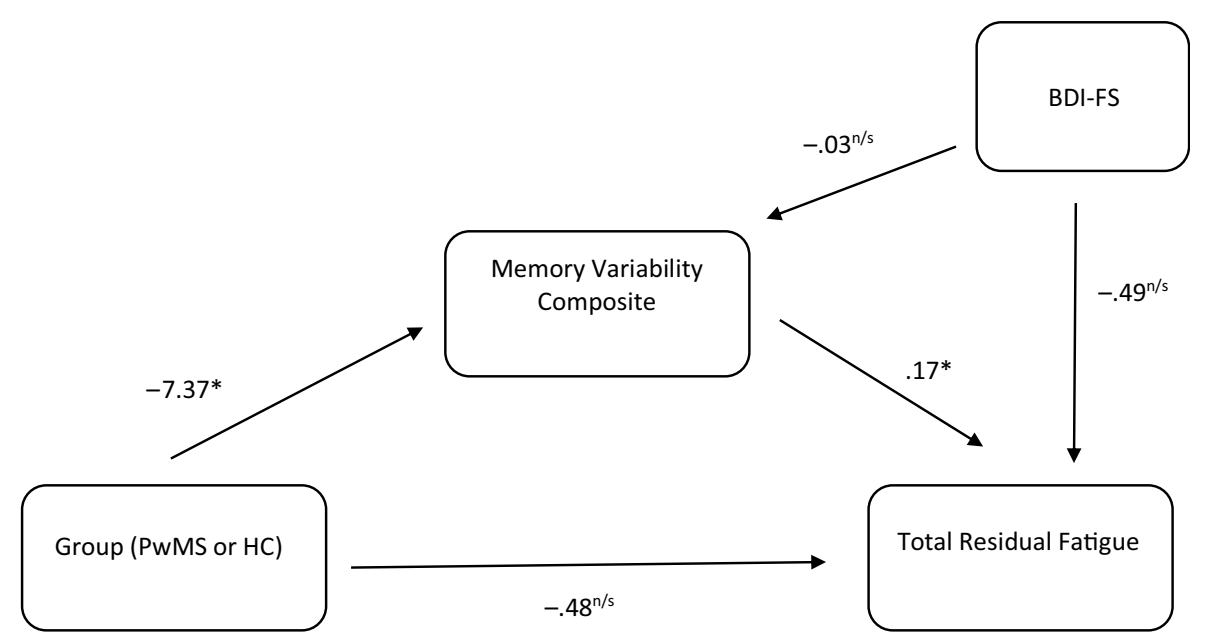

Fig. 2. Mediation model with memory variability composite as mediator and depression as covariate.

BDI-FS = Beck Depression Inventory-Fast Screen; HCs = Healthy Controls; PwMS = Persons with Multiple Sclerosis.

${ }^{*}=p<.05,{ }^{\mathrm{n} / \mathrm{s}}=p>.05$ (not significant).

with fatigue, rather than overall mean cognitive performance, as the outcome. Further in line with this previous work, we hypothesized that the direction of the relationship would be that IIV mediated total reported fatigue; that is, variable performance over time would be associated with higher self-report fatigue.

The current study explored the relationship between IIV and fatigue by utilizing a multidimensional scale to measure perceived fatigue, examining IIV as a measure of performance fatiguability, and using fatigue as the outcome of interest. Another benefit of the model used in the current study was that it accounted for total depression scores. As discussed above, depression is a common symptom in MS, is significantly correlated with fatigue, and has previously been shown to be a significant independent predictor, accounting for $31 \%$ of the variance, of MS fatigue (Strober \& Arnett, 2005). Prior work has demonstrated that greater IIV may be associated with age-related changes (Sosnoff \& Newell, 2006). Thus, to account for these differences, age was included in the mediation model by regressing age on total fatigue and then saving the unstandardized residuals (total residual fatigue) as the new outcome variable. The current mediation models allowed us to explore the effects of IIV on fatigue controlling for the contributing effects of depression on MS-related fatigue and accounting for the significant age difference.

As discussed above, depression is common in PwMS and can also have a measurable impact on cognitive functioning. In our sample, depression scores were significantly correlated with attention/processing speed IIV, but not memory IIV. A meta-analysis by McDermott and Ebmeier (2009) highlighted that prior research on depression and cognitive impairments in different domains has included inconsistent and contradictory findings. The meta-analysis revealed a significant correlation between depression severity and cognitive performance in domains of episodic memory, executive functioning, and processing speed, but not semantic or visuospatial memory (McDermott \& Ebmeier, 2009).
Further, a review of processing speed, depression, fatigue, and cognition in PwMS found that processing speed is slower in MS depressed compared to MS nondepressed individuals (Arnett et al., 1999). Taken together, attention and processing speed do seem to be slower in individuals with depression who do not have MS and in PwMS with depression. With these findings in mind, it may be that variability, rather than just mean differences in cognitive performance, highlights a unique influence of depression on this domain compared to memory.

Consistent with our hypotheses, results of two separate mediation analyses examining the mediating effect of IIV on total residual fatigue between PwMS and HC, after covarying for BDI-FS scores, revealed that IIV on both tests of attention/processing speed and memory mediated the relationship between patient status and total residual fatigue. This relationship was such that PwMS reported more total residual fatigue than HCs and that these group differences in total residual fatigue were reduced to being nonsignificant once accounting for IIV on both tests of attention/processing speed and memory. This indicates that variability in cognitive performance is associated with an increase in perceived fatigue. In short, patient status (MS or HC) leads to differences in IIV, which, in turn, results in differences in the perceived impact of fatigue. To further test the validity of our directionality hypothesis, we tested the reverse mediation, and total residual fatigue did not significantly mediate differences in IIV between the groups. That is, it does not seem that fatigue itself leads to the variability in performance over time.

There are several potential explanations for why IIV was found to be a significant mediator of the relationship between patient status and residual perceived fatigue. It may be the case that there are potential third variables, such as patterns of neural activation or attributions about fatigue symptoms, that contribute to both perceived fatigue and IIV. For example, individuals experiencing more IIV may perceive themselves as more fatigued. This aligns with models that have 
demonstrated that the way that patients interpret and respond to their symptoms or symptom attributions may increase the subjective experience of fatigue (Skerrett \& Moss-Morris, 2006; van Kessel \& Moss-Morris, 2006). A study by Skerrett and colleagues (2006) measured symptom interpretation and behavioral responses in PwMS. Results indicated that cognitive interpretations of their symptoms, including attributing a wide range of symptoms to their MS, were associated with fatigue over and above disease severity (EDSS), remission status, and mood (Skerrett \& Moss-Morris, 2006).

Alternately, brain activation of areas thought to underlie fatigue, may also be a variable that is related to both perceived fatigue and IIV. A study by DeLuca and colleagues (2008) examined the effects of sustained mental effort on patterns of brain activation between PwMS and HCs. In this study, cognitive fatigue (e.g., fatiguability) was defined as a relative increase in cerebral activation over time. Results indicated that increased activation was seen in PwMS compared to HCs and that this may be associated with the increased cognitive effort needed to adequately perform a task over a long period of time (DeLuca et al., 2008). Further, this same group found that, when faced with increased cognitive demands, neural resources may be inefficiently activated in PwMS thus resulting in increased mental or cognitive fatigue (Chen et al., 2020). Additionally, research on the pathophysiology of MS and MS fatigue has identified the involvement in immune dysregulation of the cortico-striato-thalamo-cortical loop in the generation of MS fatigue (Chalah et al., 2015). Demyelination and neurodegeneration of these brain regions may also underlie both perceived fatigue and lead to increased variability in performance.

These findings have important implications for understanding the impact of fatigue on the daily lives of PwMS and for the treatment of this fatigue. Variable performance, such as what might occur in the case of diurnal fatigue, may be reflective of fatiguability. This may be true of variable performance across a test battery, as was the case in this study, or variability in the performance of daily tasks throughout the workday that are reflective of difficulties commonly reported by PwMS. Fortunately, manualized treatments based on the cognitive behavioral therapy model of fatigue in MS have been created and a meta-analysis of these treatments demonstrated both positive short-term and long-term effects on fatigue (Induruwa, Constantinescu, \& Gran, 2012; van den Akker et al., 2016). Further, some disease modifying pharmacological treatments have demonstrated an impact on reducing the perception of fatigue in PwMS (Jongen et al., 2010; Putzki, Yaldizli, Tettenborn, $\&$ Diener, 2009). More recently, exercise interventions have also been explored and progressive resistance training has been shown to be effective at reducing fatigue in MS (Akbar et al., 2020).

This study had several limitations. First, this study design was cross-sectional, therefore fatigue and IIV were measured on the same testing day. Future studies may explore IIV and perceived fatigue in a prospective and longitudinal design to understand the relationship between cognitive variability and fatigue over time. Additionally, the test battery administered in this study was comprised mostly of test indices known to be sensitive to difficulties experienced by PwMS. However, the battery was comprehensive and included tests covering several domains of functioning including immediate and delayed visual recall, immediate and delayed verbal recall, attention, and processing speed. Additionally, the study sample, both PwMS and HCs, was predominantly White and well educated. Therefore, these findings may not replicate in more heterogeneous populations and research on more diverse populations is warranted in the future.

Overall, this study demonstrated that IIV is an objective performance measure that is related to differences in fatiguability between PwMS and HCs. PwMS experience more variability across tests of attention/processing speed and memory and this experience of variable performance may increase the perception of fatigue. While traditional measures of performance have been variably linked to perceived fatigue, IIV may better capture the self-reported difficulties of individuals with MS-related fatigue. Another important conclusion from this study is that the relationship between MS and fatigue may not be direct, but rather may be the result of the mediating factor of cognitive variability.

\section{FINANCIAL SUPPORT}

This investigation was supported (in part) by a grant to the last author from the National Multiple Sclerosis Society (PP0978). The authors thank the many neurologists in the Pennsylvania region who contributed their time to verifying MS diagnoses and ratings and of course, for the MS participants in the project. The authors also thank Megan Smith, Fiona Barwick, Joseph Beeney, and Andrae Laws for their help with various aspects of the study.

\section{CONFLICTS OF INTEREST}

Dr. Bruce is a paid part-time employee of the National Hockey League. He is a grantee of the National Multiple Sclerosis Society, has served on the Novartis unbranded Speaker's Bureau, and has received funding from Genzyme and Med-IQ. None of the other authors have conflicts of interest to report.

\section{REFERENCES}

Akbar, N., Sandroff, B.M., Wylie, G. R., Strober, L. B., Smith, A., Goverover, Y., ... Genova, H. (2020). Progressive resistance exercise training and changes in resting-state functional connectivity of the caudate in persons with multiple sclerosis and severe fatigue: A proof-of-concept study. Neuropsychological Rehabilitation, 30(1), 54-66. https://doi.org/10.1080/09602011. 2018.1449758

Aldughmi, M., Bruce, J., \& Siengsukon, C.F. (2017). Relationship between fatigability and perceived fatigue measured using the neurological fatigue index in people with multiple sclerosis. 
International Journal of MS Care, 19(5), 232-239. https://doi. org/10.7224/1537-2073.2016-059

American Psychiatric Association. (2013). Diagnostic and statistical manual of mental disorders: DSM-5. DSM-5. https://doi.org/ 10.1176/appi.books.9780890425596.744053

Arnett, P.A., Barwick, F.H., \& Beeney, J.E. (2008). Depression in multiple sclerosis: Review and theoretical proposal. Journal of the International Neuropsychological Society, 14(5), 691-724. https://doi.org/10.1017/S1355617708081174

Arnett, P.A., Higginson, C.I., Voss, W.D., Bender, W.I., Wurst, J.M., \& Tippin, J.M. (1999). Depression in multiple sclerosis: Relationship to working memory capacity. Neuropsychology, 13(4), 546-556. https://doi.org/10.1037/0894-4105.13.4.546

Arnett, P., Smith, M.M., Barwick, F.H., Benedict, R.H.B., \& Ahlstrom, B.P. (2008). Oralmotor slowing in multiple sclerosis: Relationship to neuropsychological tasks requiring an oral response. Journal of the International Neuropsychological Society, 14(3), 454-462. https://doi.org/10.1017/S1355617 708080508

Beck, A., Steer, R., \& Brown, G. (2000). BDI-Fast Screen for medical patients manual. San Antonio, TX: Psychological Corporation.

Benedict, R.H.B. (1997). Brief visuospatial memory test- revised: professional manual. Odessa, FL: Psychological Assessment Resource.

Benedict, R.H.B., Fishman, I., McClellan, M., Bakshi, R., \& Weinstock-Guttman, B. (2003). Validity of the beck depression inventory-fast screen in multiple sclerosis. Multiple Sclerosis, 9, 393-396. https://doi.org/10.1191/1352458503ms902oa

Bruce, J.M., Bruce, A.S., \& Arnett, P.A. (2010). Response variability is associated with self-reported cognitive fatigue in multiple sclerosis. Neuropsychology, 24(1), 77-83. https://doi.org/10. 1037/a0015046

Bryant, D., Chiaravalloti, N.D., \& DeLuca, J. (2004). Objective measurement of cognitive fatigue in multiple sclerosis. Rehabilitation Psychology, 49(2), 114-122. https://doi.org/10. 1037/0090-5550.49.2.114

Burton, C.L., Strauss, E., Hultsch, D.F., Moll, A., \& Hunter, M.A. (2006). Intraindividual variability as a marker of neurological dysfunction: A comparison of Alzheimer's disease and Parkinson's disease. Journal of Clinical and Experimental Neuropsychology, 28(1), 67-83. https://doi.org/10.1080/13803 390490918318

Busichio, K., Tiersky, L.A., Deluca, J., \& Natelson, B.H. (2004). Neuropsychological deficits in patients with chronic fatigue syndrome. Journal of the International Neuropsychological Society, 10(2), 278-285. https://doi.org/10.1017/S1355617704 102178

Cadden, M., \& Arnett, P. (2015). Factors associated with employment status in individuals with multiple sclerosis: Cognition, fatigue, and motor function. International Journal of MS Care, 17(6), 284-291. https://doi.org/10.7224/15372073.2014-057

Chalah, M.A., Riachi, N., Ahdab, R., Créanget, A., Lefaucheur, J.P., \& Ayache, S.S. (2015). Fatigue in multiple sclerosis: Neural correlates and the role of non-invasive brain stimulation. Frontiers in Cellular Neuroscience, 9(NOV), 1-15. https://doi.org/10.3389/ fncel.2015.00460

Charvet, L., Serafin, D., \& Krupp, L. B. (2014). Fatigue in multiple sclerosis. Fatigue: Biomedicine, Health and Behavior, 2(1), 3-13. https://doi.org/10.1080/21641846.2013.843812
Chen, M.H., Wylie, G.R., Sandroff, B.M., Dacosta-Aguayo, R., DeLuca, J., \& Genova, H.M. (2020). Neural mechanisms underlying state mental fatigue in multiple sclerosis: a pilot study. Journal of Neurology, (0123456789). https://doi.org/10.1007/ s00415-020-09853-w

Cockshell, S.J. \& Mathias, J.L. (2010). Cognitive functioning in chronic fatigue syndrome: A meta-analysis. Psychological Medicine, 40(8), 1253-1267. https://doi.org/10.1017/S0033291 709992054

Cole, V.T., Weinberger, D.R., \& Dickinson, D. (2011). Intra-individual variability across neuropsychological tasks in schizophrenia: A comparison of patients, their siblings, and healthy controls. Schizophrenia Research, 129(1), 91-93. https:// doi.org/10.1016/j.schres.2011.03.007

Delis, D.C., Kaplan, E., \& Kramer, J.H. (2001). Delis-Kaplan executive function. San Antonio, TX: Psychological Corporation.

Delis, D.C., Kramer, J.H., Kaplan, E., \& Ober, B.A. (2000). California verbal learning test- second edition. Adult Version. San Antonio, TX: Psychological Corporation.

DeLuca, J. (2005). Issues in clinic and cognitive neuropsychology. In Fatigue as a window to the brain (pp. 37-57). MIT Press.

DeLuca, J., Genova, H.M., Hillary, F.G., \& Wylie, G. (2008). Neural correlates of cognitive fatigue in multiple sclerosis using functional MRI. Journal of the Neurological Sciences, 270(1-2), 28-39. https://doi.org/10.1016/j.jns.2008.01.018

Fisk, J.D., Pontefract, A., Ritvo, P.G., Archibalnd, C.J., \& Murray, T.J. (1994). The impact of fatigue on patients with multiple scleorosis. Canadian Journal of Neurological Sciences, 21, 9-14.

Fisk, J.D., Ritvo, P.G., Ross, L., Haase, D.A., Marrie, T.J., \& Schlech, W.F. (1994). Measuring the functional impact of fatigue: Initial validation of the fatigue impact scale. Clinical Infectious Diseases, 18, S79-S83. https://doi.org/10.1093/ clinids/18.Supplement_1.S79

Freal, J.E., Kraft, G.H., \& Coryell, J.K. (1984). Symptomatic fatigue in multiple sclerosis. Archives of Physical Medicine and Rehabilitation, 65, 135-138.

Fuentes, K., Hunter, M.A., Strauss, E., \& Hultsch, D.F. (2001). Intraindividual variability in cognitive performance in persons with chronic fatigue syndrome. Clinical Neuropsychologist, 15(2), 210-227. https://doi.org/10.1076/clin.15.2.210.1896

Guty, E. \& Arnett, P. (2018). Post-concussion symptom factors and neuropsychological outcomes in collegiate athletes. Journal of the International Neuropsychological Society, 24(7), 1-9. https://doi.org/10.1017/S135561771800036X

Hanken, K., Eling, P., \& Hildebrandt, H. (2015). Is there a cognitive signature for MS-related fatigue? Multiple Sclerosis (Houndmills, Basingstoke, England), 21(4), 376-381. https://doi.org/10.1177/ 1352458514549567

Haynes, B.I., Bauermeister, S., \& Bunce, D. (2017). Does within-person variability predict errors in healthy adults aged 18-90? Quarterly Journal of Experimental Psychology, 70(8), 1722-1731. https://doi.org/10.1080/17470218.2016.120 4328

Heyanka, D.J., Holster, J.L., \& Golden, C.J. (2013). Intraindividual neuropsychological test variability in healthy individuals with high average intelligence and educational attainment. International Journal of Neuroscience, 123(8), 526-531. https://doi.org/10.3109/00207454.2013.771261

Homorodean, R., Leucuta, D.C., \& Perju-Dumbravă, L. (2016). Fatigue in multiple sclerosis. Human and Veterinary Medicine, 8(3), 148-153. 
Induruwa, I., Constantinescu, C.S., \& Gran, B. (2012). Fatigue in multiple sclerosis - A brief review. Journal of the Neurological Sciences, 323(1-2), 9-15. https://doi.org/10.1016/j.jns.2012. 08.007

Johnson, S.K., Lange, G., DeLuca, J., Korn, L.R., \& Natelson, B. (1997). The effects of fatigue on neuropsychological performance in patients with chronic fatigue syndrome, multiple scleorosis, and depression. Applied Neuropsychology, 4(3), 145-153.

Jongbloed, L. (1998). Disability income: the experiences of women with multiple sclerosis. Canadian Journal of Occupational Therapy, 65(4), 193-201. https://doi.org/10.1177/000841749806 500404

Jongen, P.J., Lehnick, D., Sanders, E., Seeldrayers, P., Fredrikson, S., Andersson, M., \& Speck, J. (2010). Health-related quality of life in relapsing remitting multiple sclerosis patients during treatment with glatiramer acetate: A prospective, observational, international, multi-centre study. Health and Quality of Life Outcomes, 8, 1-7. https://doi.org/10.1186/1477-7525-8-133

Kluger, B.M., Krupp, L.B., \& Enoka, R.M. (2013). Fatigue and fatigability in neurologic inllnesses. Neurology, 80, 409-416.

Krupp, L.B. \& Elkins, L.E. (2000). Fatigue and declines in cognitive functioning in multiple sclerosis. Neurology, 55(7), 934-939. https://doi.org/10.1212/WNL.55.7.934

Krupp, L.B., Serafin, D.J., \& Christodoulou, C. (2010). Multiple sclerosis-associated fatigue. Expert Review of Neurotherapeutics, 10(9), 1437-1447. https://doi.org/10.1586/ ern.10.99

Kujala, P., Portin, R., Revonsuo, A., \& Ruutiainen, J. (1995). Attention related performance in two cognitively different subgroups of patients with multiple sclerosis. Journal of Neurology, Neurosurgery, and Psychiatry, 59(1), 77-82. https:// doi.org/10.1136/jnnp.59.1.77

Lezak, M.D., Howieson, D.B., Bigler, E.D., \& Tranel, D. (2012). Neurospychological assessment (5th ed.). New York, NY: Oxford University Press.

MacAllister, W.S. \& Krupp, L.B. (2005). Multiple sclerosis - related fatigue. Physical Medicine and Rehabilitation Clinics of North America, 16(2), 483-502. https://doi.org/10.1016/j.pmr.2005. 01.014

MacDonald, S.W.S., Nyberg, L., \& Bäckman, L. (2006). Intra-individual variability in behavior: links to brain structure, neurotransmission and neuronal activity. Trends in Neurosciences, 29(8), 474-480. https://doi.org/10.1016/j.tins.2006.06.011

McDermott, L.M. \& Ebmeier, K.P. (2009). A meta-analysis of depression severity and cognitive function. Journal of Affective Disorders, 119(1-3), 1-8. https://doi.org/10.1016/j.jad.2009. 04.022

Merritt, V.C., Greenberg, L.S., Guty, E.T., Bradson, M.L., Rabinowitz, A.R. \& Arnett, P.A. (2019). Beyond measures of central tendency: Novel methods to examine sex differences in neuropsychological performance following sports-related concussion in collegiate athletes. Journal of the International Neuropsychological Society, 25(10), 1094-1100. https://doi. org/10.1017/S1355617719000882

Multiple Sclerosis Council for Clinical Practice Guidelines. (1998). Fatigue and multiple sclerosis: evidence-based management strategies for fatigue in multiple sclerosis. Washington, DC: Paralyzed Veterans of America.

Parmenter, B.A., Denney, D.R., \& Lynch, S.G. (2003). The cognitive performance of patients with multiple sclerosis during periods of high and low fatigue. Multiple Sclerosis, 9(2), 111-118. https:// doi.org/10.1191/1352458503ms859oa

Paul, R.H., Beatty, W.W., Schneider, R., Blanco, C.R., \& Hames, K.A. (1998). Cognitive and physical fatigue in multiple sclerosis: Relations between self-report and objective performance. Applied Neuropsychology, 5(3), 143-148. https://doi. org/10.1207/s15324826an0503_5

Pokryszko-Dragan, A., Zagrajek, M., Slotwinski, K., Bilinska, M., Gruszka, E., \& Podemski, R. (2016). Event-related potentials and cognitive performance in multiple sclerosis patients with fatigue. Neurological Sciences, 37(9), 1545-1556. https://doi.org/10. 1007/s10072-016-2622-x

Preacher, K.J. \& Hayes, A.F. (2004). SPSS and SAS procedures for estimating indirect effects in simple mediation models. Behavioral Research Methods, Instruments, and Computers, 36(4), 717-731.

Putzki, N., Yaldizli, Ö., Tettenborn, B., \& Diener, H.C. (2009). Multiple sclerosis associated fatigue during Natalizumab treatment. Journal of the Neurological Sciences, 285(1-2), 109-113. https://doi.org/10.1016/j.jns.2009.06.004

Rabinowitz, A.R. \& Arnett, P.A. (2013). Intraindividual cognitive variability before and after sports-related concussion. Neuropsychology, 27(4), 481-490. https://doi.org/10.1037/ a0033023

Rao, S. \& Cognitive Function Study Group, N. (1990). A manual for brief repeatable battery of the neuropsychological tests in multiple sclerosis. New York: National Multiple Sclerosis Society.

Riegler, K.E., Guty, E.T., \& Arnett, P.A. (2019). Neuropsychological test performance in depressed and nondepressed collegiate athletes following concussion. Neuropsychology, 34(1), 63-76. https://doi.org/10.1037/neu0000582

Robertson, I.H., Ward, T., Ridgeway, V., \& Nimmo-smith, I. (2001). Test reviews the test of everyday attention (TEA). The Application of Occupational Psychology to Employment and Disability, 4(1), 51-55.

Schwartz, C.E., Coulthard-Morris, L., \& Zeng, Q. (1996). Psychosocial correlates of fatigue in multiple sclerosis. Archives of Physical Medicine and Rehabilitation, 77(2), 165-170. https://doi.org/10.1016/S0003-9993(96)90162-8

Shum, D.H.K., McFarland, K.A., \& Bain, J.D. (1990). Construct validity of eight tests of attention: Comparison of normal and closed head injured samples. Clinical Neuropsychologist, 4(2), 151-162. https://doi.org/10.1080/13854049008401508

Skerrett, T.N. \& Moss-Morris, R. (2006). Fatigue and social impairment in multiple sclerosis: The role of patients' cognitive and behavioral responses to their symptoms. Journal of Psychosomatic Research, 61(5), 587-593. https://doi.org/10. 1016/j.jpsychores.2006.04.018

Sosnoff, J.J. \& Newell, K.M. (2006). The generalization of perceptual-motor intra-individual variability in young and old adults. The Journals of Gerontology Series B: Psychological Sciences and Social Sciences, 61(5), 304-310. https://doi.org/10.1093/ geronb/61.5.P304

Strober, L.B. \& Arnett, P.A. (2005). An examination of four models predicting fatigue in multiple sclerosis. Archives of Clinical Neuropsychology, 20(5), 631-646. https://doi.org/10.1016/j. acn.2005.04.002

Strober, L.B. \& Arnett, P.A. (2010). Assessment of depression in multiple sclerosis: development of a "Trunk and Branch" model. The Clinical Neuropsychologist, 24(7), 1146-1166. 
Strober, L.B. \& Arnett, P.A. (2015). Depression in multiple sclerosis: the utility of common self-report instruments and development of a disease-specific measure. Journal of Clinical and Experimental Neuropsychology, 37(7), 722-732. https://doi. org/10.1080/13803395.2015.1063591

Strober, L.B., Chiaravalloti, N., \& DeLuca, J. (2018). Should I stay or should I go? A prospective investigation examining individual factors impacting employment status among individuals with multiple sclerosis (MS). Work, 59(1), 39-47. https://doi.org/10. 3233/WOR-172667

The Wechsler Test of Adult Reading (WTAR): Test manual. (2001). San Antonio, TX: The Psychological Corporation.

Thompson, A.J., Banwell, B.L., Barkhof, F., Carroll, W.M., Coetzee, T., Comi, G., ... Cohen, J.A. (2018). Diagnosis of multiple sclerosis: 2017 revisions of the McDonald criteria. Lancet Neurology, 17(2), 162-173. https://doi.org/10.1016/ S1474-4422(17)30470-2

van den Akker, L.E., Beckerman, H., Collette, E.H., Eijssen, I.C.J.M., Dekker, J., \& de Groot, V. (2016). Effectiveness of cognitive behavioral therapy for the treatment of fatigue in patients with multiple sclerosis: A systematic review and meta-analysis. Journal of Psychosomatic Research, 90(2016), 33-42. https://doi.org/10.1016/j.jpsychores.2016.09.002

van Kessel, K. \& Moss-Morris, R. (2006). Understanding multiple sclerosis fatigue: A synthesis of biological and psychological factors. Journal of Psychosomatic Research, 61(5), 583-585. https:// doi.org/10.1016/j.jpsychores.2006.03.006

Wang, C., Ding, M., \& Kluger, B.M. (2014). Change in intraindividual variability over time as a key metric for defining performance-based cognitive fatigability. Brain and Cognition, 85(1), 251-258. https://doi.org/10.1016/j.bandc.2014.01.004

Weschler, D. (1997). Weschler adult intelligence scale-III. New York, NY: Psychological Corporation.

Whitehead, L. (2009). The measurement of fatigue in chronic illness: A systematic review of unidimensional and multidimensional fatigue measures. Journal of Pain and Symptom Management, 37(1), 107-128. https://doi.org/10.1016/j.jpain symman.2007.08.019

Wojtowicz, M., Berrigan, L.I., \& Fisk, J.D. (2012). Intra-individual variability as a measure of information processing difficulties in multiple sclerosis. International Journal of MS Care, 14(2), 77-83. https://doi.org/10.7224/1537-2073-14.2.77 